
\title{
$\begin{array}{ll}\text { Research Square } & \begin{array}{l}\text { They should not be considered conclusive, used to inform clinical practice, } \\ \text { or referenced by the media as validated information. }\end{array}\end{array}$ \\ Real-world Data Analysis of Immunotherapy in Advanced Lung Cancer
}

\author{
Meiling Sun \\ Qilu Hospital, Shandong University \\ Huaijun Ji \\ Weihai Municipal Hospital \\ Ning Xu \\ Weihai Municipal Hospital \\ Peng Jiang \\ Weihai Municipal Hospital

\section{Tao Qu} \\ Weihai Municipal Hospital \\ Yu Li ( $\nabla$ qlliyures@163.com ) \\ Qilu Hospital, Shandong University
}

\section{Research Article}

Keywords: Immunotherapy, Immune checkpoint inhibitor (ICI), PD-L1 inhibitor, NSCLC, Prognostic

Posted Date: December 14th, 2021

DOI: https://doi.org/10.21203/rs.3.rs-1031213/v1

License: (c) (i) This work is licensed under a Creative Commons Attribution 4.0 International License. Read Full License 


\section{Abstract}

Background

This study was designed to investigate the clinical application, efficacy, and safety of immune checkpoint inhibitors (ICls) in the treatment of lung cancer in the real world.

Methods

A retrospective, observational analysis was conducted on patients treated with ICls in four tertiary hospitals in the region from January 2015 to March 2021, to evaluate the clinical efficacy of ICl single-agent or combined chemotherapy and anti-vascular drugs in the first-line or second-line treatment of patients with advanced lung cancer.

Results

Three hundred and fifteen patients were enrolled in this study. The objective response rate (ORR) and disease control rate (DCR) were 36.5\% (115/315) and 94.0\% (296/315), respectively, the median progression-free survival (PFS) was 10.8 months, and the median overall survival (OS) was not reached. A total of 165 patients received ICl as the first-line treatment, the median treatment cycle was 8 cycles ( $2-20$ cycles), the short-term efficacy ORR was 41.2\%, DCR was $94.5 \%$, and the median PFS was 12.0 months. 150 patients received ICl treatment as second-line treatment, the median treatment cycle was five cycles (2-10 cycles), the short-term efficacy ORR was $31.3 \%$, DCR was $93.3 \%$, and the median PFS was 10.0 months. There were no statistically significant differences in ORR, DCR, or median PFS with ICl as the first-line treatment compared with the second-line treatment $(P>0.05)$. The results of subgroup analysis showed that Karnofsky performance status (KPS) score, EGFR mutation status, and number of treatment lines were not correlated with median PFS((P>0.05). However, there were statistically significant differences in programmed death-ligand 1(PD-L1) expression, pathological types, hormone interference, and antibiotic (Abx) treatment among all groups $(P<0.05)$. In terms of safety, the overall incidence of adverse reactions in 315 patients was $62.5 \%$, and the incidence of immune-related adverse events(irAEs) was $13.7 \%$. Grade $1-2$ and 3-4 incidence of adverse events were $34.9 \%$ and $27.65 \%$, respectively. There were four patients who experienced fatal irAEs, two cases were liver damage leading to liver failure, one case was immune related pneumonia, and one case was immune related myocarditis.

Conclusion

In the real world, immunotherapy has a good effect on patients with advanced lung cancer and significantly improves ORR and PFS.

\section{Background}

Lung cancer remains the malignant tumor with the highest morbidity and mortality in China and the world, among which non-small cell lung cancer (NSCLC) accounts for approximately $85 \%$ of cases [1]. Due to the lack of specific clinical symptoms in the early stage of lung cancer, most patients are already at an advanced stage when they are diagnosed, which delays the diagnosis and treatment and leads to poor prognosis. For the traditional treatment model, the 5-year survival rate of advanced lung cancer is only approximately $5 \%$, and after first-line antitumor treatment, the disease is still not under control, and metastasis occurs in some patients [2]. In recent years, with the 
continuous advancement of the diagnosis and treatment of lung cancer, the treatment mode of lung cancer has gradually changed from the empirical single mode to the refined comprehensive diagnosis and treatment mode based on the genomic characteristics of patients. By selectively inhibiting the programmed cell death-1 (PD-1)/PDL1 pathway, ICls activate the immune activity of T lymphocytes and resume the antitumor immune response of the body, and using the body's own immune system plays a role in killing tumor cells [3]. Immunotherapy, represented by ICls, has changed the treatment mode of a variety of advanced malignant tumors, especially in the field of lung cancer treatment, which has played an important role in the long-term survival effect of some patients with advanced lung cancer and greatly improved the prognosis of patients with lung cancer.

Based on the results of CheckMate 024, the Food and Drug Administration (FDA) approved pembrolizumab combined with chemotherapy for the first-line treatment of metastatic NSCLC with PD-L1(TPS $\geq 50 \%$ ), which also highlights the importance of PD-L1 detection[4]. Randomized clinical trial results from CheckMate 057[5], KEYNOTE-001[6] and KEYNOTE-010[7] showed that nivolumab, atezolizumab, and pembrolizumab were superior to chemotherapy in ORR, PFS, and OS. For nondriver gene mutation/fusion advanced NSCLC with PD-L1 expression $<50 \%$, the results of KEYNOTE-189[8], IMpower-150[9], and other trials have shown that ICl combined with chemotherapy could bring more survival benefits to patients than chemotherapy. Although the curative effect is considerable, the overall effective response rate is low, and some serious irAEs and other problems may accelerate disease progression and even be life-threatening, which limits the clinical application of ICls.

In recent years, immunotherapy has developed rapidly in the treatment of lung cancer. To date, there are eight types of immune drugs on the market in China, including the domestic drugs tislelizumab, sintilimab, camrelizumab, and toripalimab. Imported drugs include pembrolizumab, nivolumab, durvalumab, and atezolizumab, of which tislelizumab and sintilimab are not approved for lung cancer indications. In 2018, the National Comprehensive Cancer Network (NCCN) recommended pembrolizumab in combination with chemotherapy plus bevacizumab for the first-line treatment of advanced nonsquamous NSCLC patients without EGFR or ALK gene mutations, with the recommended level being grade I. Based on the phase III clinical study of KEYNOTE-407, NCCN guidelines recommend the PD-1 inhibitor pembrolizumab as the first-line treatment for patients with PD-L1 $\geq 50 \%$ and driver gene-negative advanced NSCLC in 2019. In the latest Chinese Society of Clinical Oncology(CSCO) guidelines for the diagnosis and treatment of nonsmall cell lung cancer in 2020, in the

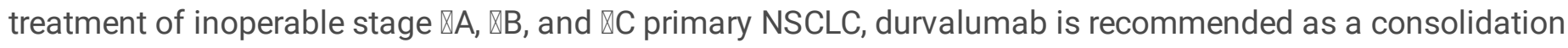
treatment after concurrent chemoradiation to grade I. For the first-line treatment of stage IV NSCLC without driver gene mutations, pembrolizumab single-agent first-line therapy(limited to PD-L1 TPS $\geq 50 \%$ ), pembrolizumab combined with pemetrexed and platinum are upregulated to level $₫$ Recommendation (Class $1 \mathrm{~A}$ evidence). Additional single-agent first-line treatment occurs with pembrolizumab monotherapy (PD-L1 TPS is 1\% 49\%) (Class 2A evidence). Immunotherapy in Level $\triangle$ Recommendation is newly added domestic PD-1 inhibitor camrelizumab combined with pemetrexed and platinum (Class $1 \mathrm{~A}$ evidence). Atezolizumab combined with paclitaxel plus carboplatin plus bevacizumab first-line treatment was revised up from grade $₫$ recommendation to grade $\otimes$ recommendation (Class $1 \mathrm{~A}$ evidence). Atezolizumab combined with albumin paclitaxel plus carboplatin was newly added as first-line therapy (Class $1 \mathrm{~A}$ evidence). Second-line treatment of NSCLC without driver gene mutations in stage $\triangle$ consists of: the second-line treatment of atezolizumab immune monotherapy upgraded from grade $\nabla$ recommendation to grade $₫$ recommendation (Class $1 \mathrm{~A}$ evidence), which is based on the results of OAK study subgroup analysis[10-11]. The 2020 CSCO Guidelines for the Diagnosis and Treatment recommend atezolizumab combined with EC as a Class I recommendation for extensive-stage small cell lung cancer (SCLC). For the third-line and later-line treatment of relapsed SCLC, nivolumab and pembrolizumab are recommended as 
grade II. Since durvalumab combined with chemotherapy for the treatment of extensive-stage SCLC has not yet been approved in China, the guidelines put durvalumab combined with chemotherapy as the level III recommendation for extensive-stage SCLC [12].

This study collected the case data of 315 patients with advanced lung cancer who received immunotherapy in four hospitals in Shandong from January 2015 to March 2021, summarized the clinical characteristics, diagnosis and treatment, curative effect and adverse reactions, and aimed to explore the clinical efficacy and safety of ICls in first-line or second-line treatment of advanced lung cancer patients in the real world and possible factors related to efficacy and side effects.

\section{Methods}

\section{Data collection}

We retrospectively collected patients with advanced lung cancer who received immunotherapy in a number of hospitals in Shandong from January 2015 to March 2021. The patients' age, sex, past history, smoking history, pathological type, KPS score, TNM staging, tumor metastasis (brain/bone/liver metastasis), driver gene detection, PD-L1 expression, hormone or Abx interference were collected. Treatment included immunotherapy drugs and methods, number of immunotherapy lines (1 line/2 lines), clinical efficacy, and adverse reactions.

\section{Patient selection}

(1) Inclusion criteria: KPS score>50 points, estimated survival time>3 months; pathological or cytological diagnosis of lung cancer; and at least two cycles of ICI treatment. (2) Exclusion criteria: no clear pathological diagnosis information; patients with other tumors at the same time; KPS score $\leq 50$ points; no efficacy evaluation after ICI treatment; patients with important organ dysfunction; (3) Hormonal interference was defined as receiving no less than $10 \mathrm{mg}$ of prednisone equivalent for more than 20 days from the first day of ICI treatment. (4) Evaluation of Abx treatment status: Patients who received Abx within 1 month before or after the first administration of ICI were defined as the Abx treatment group.

\section{Efficacy assessment}

According to the Response Evaluation Criteria in Solid Tumors (RECIST) version 1.1; efficacy, prognostic evaluation, and adverse reaction grading were evaluated. The evaluation was based on imaging examinations, and efficacy evaluation was performed at least once every two courses. An overall response was defined as a complete response $(C R)$, partial response $(P R)$, stable disease, or progressive disease. ORR $=(C R+P R) /(C R+P R+S D+P D)$ $\times 100 \%$; $D C R=(C R+P R+S D) /(C R+P R+S D+P D) \times 100 \%$; PFS was defined as the time from the start of immunotherapy initiation to the first event (tumor progression or death from any cause). OS was defined as the time from the start of immunotherapy to death from any cause. At each patient review, safety assessment was carried out through laboratory-related tests. Blood tests included routine blood tests, biochemistry, and hormone measurements. IrAEs are judged according to version 4.03 of the National Cancer Institute Common Terminology Criteria for Adverse Events (NCI-CTCAE) and are divided into grades I-V. In this study, grades III-IV were defined as moderate to severe adverse reactions, and grade $\mathrm{V}$ was defined as death.

\section{Follow-up}


The patients were followed up by consulting outpatient records, hospitalization medical records, and telephone inquiries. The last follow-up date was March 31, 2021. The content of the follow-up included the general condition of the patient, tumor treatment, disease progression, and treatment side effects. Those who were lost to follow-up and those who did not die were treated according to the cutoff value, and the cutoff time was the last follow-up time at which they were confirmed not dead. If the follow-up was lost, the time of the last follow-up was recorded.

\section{Statistical analysis}

All statistical analyses and graphing were performed with SPSS ver. 23.0 and GraphPad Prism 7 software. All survival analyses were estimated using Kaplan-Meier curves and compared using the log-rank test. The Cox proportional hazards model was used for multivariate analyses of PFS. P<0.05 was considered statistically significant.

\section{Ethical statement}

This program was performed in accordance with the principles of good clinical practice and was approved by the institutional review board of each hospital. All patients provided written informed consent before participation.

\section{Results}

\section{Baseline characteristics}

From January 2015 to March 2021, a total of 400 patients were screened, and 315 lung cancer patients who met the criteria were included. The baseline demographic and clinical characteristics are shown in Table 1.

The median age of all patients was 63 years (range 27-85), including 254 males (80.6\%) and 61 females (19.4\%). A total of 216 patients were smokers $(68.6 \%)$, and 285 patients $(90.5 \%)$ had a KPS score $\geq 90$ at the time of diagnosis. By histology, 135 patients (42.9\%) had adenocarcinoma, 117 (37.1\%) had squamous cell carcinoma, and most patients (83.8\%) had stage IV disease. Distant metastasis was identified in 158 patients (50.2\%), and the most common site of metastasis was bone (22.5\%), followed by brain (24.4\%) and liver (10.5\%). Of the patients undergoing genetic testing, only 74 patients (23.5\%) carried genetic mutations. In terms of PD-L1 expression level, 67 cases $(21.3 \%)$ were $\geq 50 \%, 77$ cases $(24.4 \%)$ were $1-49 \%$, and 109 cases $(34.6 \%)$ were less than $1 \%$. Thirteen patients (4.1\%) were treated with hormones for more than 20 days, and 302 patients $(95.9 \%)$ had no hormone interference. Forty-three patients (13.7\%) received Abx treatment for more than 7 days, and 48 patients (15.2\%) received Abx for less than 7 days. A total of 165 patients (52.4\%) received ICls as the first-line application, while 150 patients (47.6\%) received ICls as the second-line application. A total of 315 patients received seven types of ICls in the past; the most used was sintilimab in 108 cases (34.3\%), and the least used was durvalumab in 16 cases $(5.1 \%)$. (Table 1$)$

\section{Table 1. Baseline characteristics of all patients}




\begin{tabular}{|c|c|c|}
\hline Characteristic & $\mathbf{N}$ & Percentage \\
\hline \multicolumn{3}{|l|}{ Sex } \\
\hline Male & 254 & 80.6 \\
\hline Female & 61 & 19.4 \\
\hline \multicolumn{3}{|l|}{ Comorbidities } \\
\hline Hypertension & 105 & 33.3 \\
\hline Diabetes mellitus & 46 & 14.6 \\
\hline Coronary heart disease & 37 & 11.7 \\
\hline \multicolumn{3}{|l|}{ Smoking history } \\
\hline Yes & 216 & 68.6 \\
\hline No & 99 & 31.4 \\
\hline \multicolumn{3}{|c|}{ KPS score at the time of diagnosis } \\
\hline$\geq 90$ & 285 & 90.5 \\
\hline$\nabla 90$ & 30 & 9.5 \\
\hline \multicolumn{3}{|l|}{ Histology } \\
\hline Adenocarcinoma & 135 & 42.9 \\
\hline Squamous cell carcinoma & 117 & 37.1 \\
\hline Small cell carcinoma & 45 & 14.3 \\
\hline Large cell carcinoma & 6 & 1.9 \\
\hline Unknown & 12 & 3.8 \\
\hline \multicolumn{3}{|l|}{ Clinical stage } \\
\hline$\nabla-\nabla$ & 7 & 2.2 \\
\hline$\square$ & 44 & 14.0 \\
\hline प & 264 & 83.8 \\
\hline \multicolumn{3}{|l|}{ Metastasis site } \\
\hline Brain & 54 & 17.1 \\
\hline Bone & 71 & 22.5 \\
\hline Liver & 33 & 10.5 \\
\hline \multicolumn{3}{|l|}{ Mutational status } \\
\hline EGFR & 59 & 18.7 \\
\hline ALK & 15 & 4.8 \\
\hline Negative & 79 & 25.1 \\
\hline
\end{tabular}




\begin{tabular}{|c|c|c|}
\hline Unknown & 162 & 51.4 \\
\hline \multicolumn{3}{|c|}{ PD-L1 expression levels } \\
\hline$\nabla 1 \%$ & 109 & 34.6 \\
\hline $1-49 \%$ & 77 & 24.4 \\
\hline$\geq 50 \%$ & 67 & 21.3 \\
\hline Unknown & 62 & 19.7 \\
\hline \multicolumn{3}{|c|}{ Hormone interference } \\
\hline Yes & 13 & 4.1 \\
\hline No & 302 & 95.9 \\
\hline \multicolumn{3}{|l|}{ Abx treatment } \\
\hline 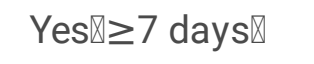 & 43 & 13.7 \\
\hline Yes $\triangle \otimes 7$ days $\bigotimes$ & 48 & 15.2 \\
\hline No & 224 & 71.1 \\
\hline \multicolumn{3}{|l|}{ Lines of therapy } \\
\hline 1 & 165 & 52.4 \\
\hline 2 & 150 & 47.6 \\
\hline \multicolumn{3}{|l|}{ Immune drugs } \\
\hline atezolizumab & 20 & 6.3 \\
\hline durvalumab & 16 & 5.1 \\
\hline sintilimab & 108 & 34.3 \\
\hline pembrolizumab & 38 & 12.1 \\
\hline camrelizumab & 46 & 14.6 \\
\hline tislelizumab & 59 & 18.7 \\
\hline toripalimab & 28 & 8.9 \\
\hline
\end{tabular}

\section{Treatment plan and treatment cycle}

All 315 patients received two or more cycles of $\mathrm{ICl}$. 165 patients received with $\mathrm{ICl}$ as the first-line treatment, 26 received $I C I$ combined with chemotherapy plus anti-angiogenic therapy (A1), 109 received ICI plus chemotherapy (A2), 11 received $I C I$ plus anti-angiogenic therapy (A3), 19 patients received $I C I$ alone (A4). The median treatment cycle of first-line treatment was eight cycles (2-20 cycles); 150 patients received ICI treatment as second-line treatment, 24 received $\mathrm{ICl}$ combined with chemotherapy plus anti-angiogenic therapy (B1), 60 received ICI plus chemotherapy (B2), 33 received ICI plus anti-angiogenic therapy (B3), 33 patients received ICI alone (B4). The median treatment cycle of second-line treatment was five cycles (2-10 cycles). (Table 2) 
Table 2. Correlations between the clinical features of different treatment modalities with ORR and PFS 


\begin{tabular}{|c|c|c|c|c|c|c|c|c|c|c|c|}
\hline \multirow{2}{*}{$\begin{array}{l}\text { First-line }[n=165 \square \\
\text { Treatment } \\
\text { pattern }\end{array}$} & \multirow[t]{2}{*}{$\mathrm{n}$} & & \multirow[t]{2}{*}{ R } & \multirow[t]{2}{*}{ PR } & \multirow{2}{*}{\multicolumn{2}{|c|}{ SD }} & \multirow[t]{2}{*}{ PD } & ORRप\%口 & \multirow[t]{2}{*}{ DCRD\%ם } & \multirow[t]{2}{*}{$\begin{array}{l}\text { Median } \\
\text { PFS(months) }\end{array}$} & $\mathbf{P}$ \\
\hline & & & & & & & & & & & \\
\hline $\mathrm{A} 1$ & 26 & 0 & 8 & 15 & 5 & 3 & 30.8 & 88.5 & 9.8 & 0.531 & \\
\hline $\mathrm{A} 2$ & 109 & 0 & 48 & 57 & 7 & 4 & 44.0 & 96.3 & 12 & & \\
\hline A3 & 11 & 0 & 4 & 6 & & 1 & 36.4 & 90.9 & 10.2 & & \\
\hline A4 & 19 & 0 & 8 & 10 & 0 & 1 & 42.1 & 94.7 & 16.1 & & \\
\hline \multicolumn{12}{|l|}{ Efficacy } \\
\hline 2-cycle & 165 & 0 & 68 & 88 & 8 & 9 & 41.2 & 94.5 & 12.0 & & \\
\hline 4-cycle & 102 & 0 & 45 & 50 & 0 & 7 & 44.1 & 93.1 & & & \\
\hline \multicolumn{12}{|l|}{ Histology } \\
\hline Adenocarcinoma & 69 & 0 & 30 & 34 & 4 & 5 & 43.5 & 92.8 & 16.0 & 0.015 & \\
\hline $\begin{array}{l}\text { Squamous cell } \\
\text { carcinoma }\end{array}$ & 68 & 0 & 26 & 41 & 1 & 1 & 38.2 & 98.5 & 13.6 & & \\
\hline $\begin{array}{l}\text { Small cell } \\
\text { carcinoma }\end{array}$ & 17 & 0 & 9 & 6 & & 2 & 52.9 & 88.2 & 6.6 & & \\
\hline \multicolumn{12}{|l|}{$\begin{array}{l}\text { Mutational } \\
\text { status }\end{array}$} \\
\hline EGFR & 32 & 0 & 12 & 18 & 8 & 2 & 37.5 & 93.8 & 12 & 0.568 & \\
\hline ALK & 7 & 0 & 3 & 4 & & 0 & 42.9 & 100 & $\begin{array}{l}\text { not } \\
\text { reached }\end{array}$ & & \\
\hline Negative & 32 & 0 & 16 & 14 & 4 & 2 & 50 & 93.8 & 16 & & \\
\hline Unknown & 94 & 0 & 37 & 52 & 2 & 5 & 39.4 & 94.7 & 11.4 & & \\
\hline \multicolumn{12}{|l|}{$\begin{array}{l}\text { PD-L1 } \\
\text { expression levels }\end{array}$} \\
\hline$\otimes 1 \%$ & 51 & 0 & 15 & 29 & 9 & 7 & 29.4 & 86.3 & 6.6 & 0.000 & \\
\hline $1-49 \%$ & 44 & 0 & 19 & 23 & 3 & 2 & 43.2 & 95.5 & 12 & & \\
\hline$\geq 50 \%$ & 37 & 0 & 22 & 15 & 5 & 0 & 59.5 & 100 & $\begin{array}{l}\text { not } \\
\text { reached }\end{array}$ & & \\
\hline Unknown & 33 & 0 & 12 & 21 & 1 & 0 & 36.4 & 100 & 10.3 & & \\
\hline \multicolumn{12}{|l|}{$\begin{array}{l}\text { Hormone } \\
\text { interference }\end{array}$} \\
\hline Yes & 7 & 0 & 3 & 3 & & 1 & 42.9 & 85.7 & $\begin{array}{l}\text { not } \\
\text { reached }\end{array}$ & 0.573 & \\
\hline No & 158 & 0 & 65 & 85 & 5 & 8 & 41.1 & 94.9 & 12.0 & & \\
\hline
\end{tabular}


Abx treatment

\begin{tabular}{|c|c|c|c|c|c|c|c|c|c|}
\hline 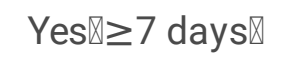 & 26 & 0 & 7 & 14 & 5 & 26.9 & 80.8 & 4 & 0.000 \\
\hline Yes $\mathbb{Q 7}$ days $\mathbb{Z}$ & 23 & 0 & 7 & 16 & 0 & 30.4 & 100 & 10.5 & \\
\hline No & 116 & 0 & 54 & 58 & 4 & 46.6 & 96.6 & 14.3 & \\
\hline \multicolumn{10}{|l|}{$\begin{array}{l}\text { Immune drugs in } \\
\text { NSCLC }\end{array}$} \\
\hline atezolizumab & 1 & 0 & 0 & 1 & 0 & 0 & 100 & $\begin{array}{l}\text { not } \\
\text { reached }\end{array}$ & 0.032 \\
\hline durvalumab & 4 & 0 & 3 & 1 & 0 & 75.0 & 100 & $\begin{array}{l}\text { not } \\
\text { reached }\end{array}$ & \\
\hline sintilimab & 48 & 0 & 16 & 32 & 0 & 33.3 & 100 & 9.8 & \\
\hline pembrolizumab & 22 & 0 & 11 & 10 & 1 & 50.0 & 95.5 & 18.7 & \\
\hline camrelizumab & 25 & 0 & 11 & 10 & 4 & 44.0 & 84.0 & 16.1 & \\
\hline tislelizumab & 34 & 0 & 15 & 17 & 2 & 44.1 & 94.1 & 10.0 & \\
\hline toripalimab & 14 & 0 & 3 & 11 & 0 & 21.4 & 100 & 9.9 & \\
\hline
\end{tabular}

\section{Second-line}

In=165!

\section{Treatment}

pattern

$\begin{array}{llllllllll}\text { B1 } & 24 & 0 & 10 & 13 & 1 & 41.7 & 95.8 & 12.7 & 0.204 \\ \text { B2 } & 60 & 0 & 16 & 42 & 2 & 26.7 & 96.7 & 11.2 & \\ \text { B3 } & 33 & 0 & 13 & 17 & 3 & 39.3 & 90.9 & 6.5 & \\ \text { B4 } & 33 & 0 & 8 & 21 & 4 & 24.2 & 87.9 & 10\end{array}$

\section{Efficacy}

$\begin{array}{lllllllll}\text { 2-cycle } & 150 & 0 & 47 & 93 & 10 & 31.3 & 93.3 & 10.0 \\ \text { 4-cycle } & 91 & 0 & 20 & 60 & 11 & 22 & 87.9 & \end{array}$

\section{Histology}

$\begin{array}{llllllllll}\text { Adenocarcinoma } & 66 & 0 & 19 & 38 & 9 & 28.8 & 86.4 & 10 & 0.194 \\ \begin{array}{l}\text { Squamous cell } \\ \text { carcinoma }\end{array} & 49 & 0 & 17 & 31 & 1 & 34.7 & 98.0 & 18.1 & \\ \begin{array}{l}\text { Small cell } \\ \text { carcinoma }\end{array} & 28 & 0 & 9 & 19 & 0 & 32.1 & 100 & 7.4 & \end{array}$

\section{Mutational}

status

$\begin{array}{llllllllll}\text { EGFR } & 27 & 0 & 5 & 16 & 6 & 18.5 & 77.8 & 8 & 0.328 \\ \text { ALK } & 8 & 0 & 5 & 3 & 0 & 62.5 & 100 & 6.3 & \\ & & & & & & \text { Page } 10 / 23 & & \end{array}$




\begin{tabular}{|c|c|c|c|c|c|c|c|c|c|}
\hline Negative & 47 & 0 & 12 & 32 & 3 & 25.5 & 93.6 & 12.7 & \\
\hline Unknown & 68 & 0 & 25 & 42 & 1 & 36.8 & 98.5 & 10 & \\
\hline \multicolumn{10}{|l|}{$\begin{array}{l}\text { PD-L1 } \\
\text { expression levels }\end{array}$} \\
\hline$\nabla 1 \%$ & 58 & 0 & 19 & 33 & 6 & 32.8 & 89.7 & 7.4 & 0.014 \\
\hline $1-49 \%$ & 33 & 0 & 6 & 25 & 2 & 18.2 & 93.9 & 13.4 & \\
\hline$\geq 50 \%$ & 30 & 0 & 13 & 16 & 1 & 43.3 & 96.7 & $\begin{array}{l}\text { not } \\
\text { reached }\end{array}$ & \\
\hline Unknown & 29 & 0 & 9 & 19 & 1 & 31.0 & 96.6 & 6.7 & \\
\hline \multicolumn{10}{|l|}{$\begin{array}{l}\text { Hormone } \\
\text { interference }\end{array}$} \\
\hline Yes & 6 & 0 & 2 & 3 & 1 & 33.3 & 83.3 & 2.5 & 0.001 \\
\hline No & 144 & 0 & 45 & 90 & 9 & 31.3 & 93.8 & 10 & \\
\hline \multicolumn{10}{|l|}{ Abx treatment } \\
\hline Yes $\nabla \geq 7$ days $\mathbb{Z}$ & 17 & 0 & 4 & 10 & 3 & 23.5 & 82.4 & 6 & 0.001 \\
\hline 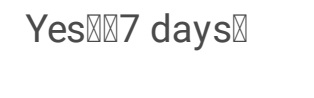 & 25 & 0 & 9 & 16 & 0 & 36.0 & 100 & $\begin{array}{l}\text { not } \\
\text { reached }\end{array}$ & \\
\hline No & 108 & 0 & 34 & 67 & 7 & 31.5 & 93.5 & 10.8 & \\
\hline \multicolumn{10}{|l|}{$\begin{array}{l}\text { Immune drugs in } \\
\text { NSCLC }\end{array}$} \\
\hline atezolizumab & 7 & 0 & 2 & 5 & 0 & 28.6 & 100 & 8.0 & 0.895 \\
\hline durvalumab & 5 & 0 & 0 & 5 & 0 & 0 & 100 & 9.7 & \\
\hline sintilimab & 48 & 0 & 14 & 32 & 2 & 29.2 & 95.8 & 13.4 & \\
\hline pembrolizumab & 15 & 0 & 10 & 3 & 2 & 66.7 & 86.7 & 10.0 & \\
\hline camrelizumab & 17 & 0 & 1 & 14 & 2 & 5.9 & 88.2 & 12.7 & \\
\hline tislelizumab & 16 & 0 & 4 & 11 & 1 & 25.0 & 93.8 & $\begin{array}{l}\text { not } \\
\text { reached }\end{array}$ & \\
\hline toripalimab & 14 & 0 & 7 & 4 & 3 & 50 & 78.6 & 8.5 & \\
\hline
\end{tabular}

\section{Efficacy}

We mainly observed short-term efficacy (2 cycles of treatment); 115 patients achieved PR, 181 patients presented SD, and the ORR and DCR of 315 patients were $36.5 \%$ (115/315) and 94.0\% (296/315), respectively. The ORRs of $\mathrm{ICl}$ as the first-line and second-line treatment were $41.2 \%(68 / 165)$ and 31.3\% (47/150), and the DCRs were $94.5 \%$ (156/165) and 93.3\% (143/150), respectively. (Table 2, Fig. 1) 
In the first-line treatment, the median PFS in Groups A1, A2, A3, and A4 was 9.8 months, 12 months, 10.2 months, and 16.1 months, respectively $(P>0.05)$. For adenocarcinoma and squamous cell carcinoma compared with SCLC, the difference in median PFS was statistically significant, which was 16.0 months, 13.6 months, and 6.6 months, respectively $(P<0.05)$. The median PFS, ORR, and DCR with ALK mutations were better than those with EGFR mutations, probably because the number of patients with ALK mutations was too small. In terms of PD-L1 expression, high PD-L1 expression compared with low PD-L1 expression prolonged the median PFS, and the differences in median PFS were statistically significant $(P<0.05)$. During the first-line application of ICI treatment, no significant difference was found in median PFS between patients with and without hormone interference $(P>0.05)$, while we observed a statistically significant improvement in the median PFS of 4 months in patients with Abx treatment $>7$ days, 10.5 months in patients with Abx treatment $<7$ days, and 14.3 months in patients without Abx treatment $(P>0.05)$.

\section{Analysis of the curative effect of second-line treatment}

In the second-line treatment, the median PFS in Groups B1, B2, B3, and B4 was 12.7 months, 11.2 months, 6.5 months, and 10 months, respectively $(P>0.05)$. There was no correlation between pathological type, gene mutation type, and median PFS in lung cancer patients ( $P>0.05)$. In terms of PD-L1 expression, the median PFS of $\otimes 1 \%, 1$ $49 \%, \geq 50 \%$ and undetected group was 7.4 months, 13.4 months, failed to reach and 6.7 months, respectively. The differences were statistically significant $(P<0.05)$. In terms of the effects of hormones and Abx, we also found significant differences in median PFS $(\mathrm{P}<0.05)$.

\section{Subgroup analysis}

The results of subgroup analysis were mainly to analyze the short-term efficacy, and the median PFS also provided evidence for subgroup differences. The KPS score, EGFR mutations, and number of treatment lines were not correlated with the median PFS ( $>0.05)$. In terms of PD-L1 expression, pathological type, hormone interference and $A b x$ treatment, the differences between the groups were statistically significant $(P<0.05)$. PD-L1 expression was $\geq 50 \%$ compared with $1-49 \%$ and $<1 \%$, and the median PFS was longer (not reaching vs. 13.4 months vs. 7.4 months). Adenocarcinoma had a more prolonged median PFS than squamous cell carcinoma and SCLC (16.0 Months vs. 12 months vs. 7.0 months), no hormone interference was longer than with hormones ( $\geq 20$ days) in median PFS (11.2 months vs. 9.8 months), and the group without Abx treatment had a longer median PFS compared with Abx treatment for $<7$ days and $\geq 7$ days (13.4 months vs. 12.0 months vs. 5.0 months). (Table 3 )

\section{Table 3. Univariate analysis of the correlation between clinical features and PFS}




\begin{tabular}{|c|c|c|c|}
\hline Variable & $\mathbf{N}$ & Median PFS(months) & $\mathbf{P}$ \\
\hline \multicolumn{4}{|c|}{ KPS score at the time of diagnosis } \\
\hline$\geq 90$ & 285 & 10.8 & 0.137 \\
\hline$\varangle 90$ & 30 & 10.5 & \\
\hline \multicolumn{4}{|l|}{ EGFR mutations } \\
\hline Yes & 59 & 10.8 & 0.153 \\
\hline No & 256 & 10.5 & \\
\hline \multicolumn{4}{|l|}{ PD-L1 expression levels } \\
\hline$\otimes 1 \%$ & 109 & 7.4 & 0.000 \\
\hline $1-49 \%$ & 77 & 13.4 & \\
\hline$\geq 50 \%$ & 67 & not reached & \\
\hline Unknown & 62 & 9.1 & \\
\hline \multicolumn{4}{|l|}{ Histology } \\
\hline Adenocarcinoma & 135 & 16.0 & 0.032 \\
\hline Squamous cell carcinoma & 117 & 12.0 & \\
\hline Small cell carcinoma & 45 & 7.0 & \\
\hline \multicolumn{4}{|l|}{ Hormone interference } \\
\hline Yes & 13 & 9.8 & 0.007 \\
\hline No & 302 & 11.2 & \\
\hline \multicolumn{4}{|l|}{ Abx treatment } \\
\hline Yes $\mathbb{Z} \geq 7$ days $\$ & 43 & 5.0 & 0.000 \\
\hline Yesه内7 days $\rrbracket$ & 48 & 12 & \\
\hline No & 224 & 13.4 & \\
\hline \multicolumn{4}{|l|}{ Lines of therapy } \\
\hline 1 & 165 & 12.0 & 0.270 \\
\hline 2 & 150 & 10.0 & \\
\hline
\end{tabular}

\section{Adverse events}

The overall incidence of adverse events in 315 patients was $62.5 \%$ (197/315), the incidence of irAEs was $13.7 \%$ (43/315), and grade $1-2$ and grade $3-4$ adverse events were $34.9 \%$ and $27.6 \%$, respectively. The most common adverse reactions were leukopenia (54.9\%), anemia (41.6\%), and fatigue (32.4\%), which did not have much effect on the progress of treatment. There were four patients with grade 5 irAEs that eventually resulted in death: two cases had liver damage leading to liver failure, one case had immune-related pneumonia, and one case had immune-related myocarditis. (Table 4) 
Table 4. Treatment-related adverse events

\begin{tabular}{|c|c|c|c|c|}
\hline Adverse events & $\underset{I-I I}{\text { Grade }}$ & $\begin{array}{l}\text { Grade } \\
\text { III-IV }\end{array}$ & $\mathrm{V}^{\text {Grade }}$ & Percentage \\
\hline Fatigue & 102 & 0 & 0 & 32.4 \\
\hline Nausea & 25 & 2 & 0 & 8.6 \\
\hline Diarrhoea & 37 & 1 & 0 & 12.1 \\
\hline Skin rash & 10 & 0 & 0 & 3.2 \\
\hline $\begin{array}{l}\text { Glu Glutamic oxaloacetic transaminase/Alanine } \\
\text { aminotransferase elevation }\end{array}$ & 15 & 3 & 2 & 5.7 \\
\hline Renal dysfunction & 8 & 0 & 0 & 2.5 \\
\hline Leucopenia & 93 & 80 & 0 & 54.9 \\
\hline Anaemia & 86 & 45 & 0 & 41.6 \\
\hline Thrombocytopenia & 31 & 18 & 0 & 15.6 \\
\hline Pneumonitis & 25 & 10 & 1 & 11.1 \\
\hline Myocarditis & 8 & 2 & 1 & 3.5 \\
\hline Endocrine toxicity & 3 & 0 & 0 & 1 \\
\hline
\end{tabular}

\section{Prognosis}

As of the last follow-up time on March 31, 2021, a total of 15 patients were lost to follow-up, with a loss rate of 4.8\%. The median PFS was 10.8 months, and the median OS was not reached. Fifty-six patients died, of which 52 patients died of tumor progression, and four patients died of severe irAEs. The one-year survival rate was $93.3 \%$. By the end of the last follow-up time, 80 patients had discontinued immunotherapy due to disease progression or side effects, while 164 patients were still receiving immunotherapy with stable conditions.

\section{Discussion}

At present, immunotherapy is regarded as a new revolution in the treatment of malignant tumors, opening up a new therapeutic field for patients with advanced lung cancer. The PD-1 inhibitors nivolumab and pembrolizumab, as well as the PD-L1 inhibitors atezolizumab and durvalumab, have been widely used in clinical practice. Nivolizumab, pembrolizumab, and atezolizumab have been approved by the FDA for advanced NSCLC [13]. With the continuous emergence of new ICls, they are expected to be used in the treatment of more malignant tumors in the future and provide better choices for cancer patients.

In recent years, a number of clinical trials have confirmed that compared with chemotherapy, immunotherapy has a significant survival advantage and has become the new standard treatment for advanced lung cancer. To the best of our knowledge, Arrieta $\mathrm{O}$ et al. published in JAMA Oncology the first prospective randomized clinical trial of pembrolizumab combined with docetaxel in the second-line treatment of advanced NSCLC patients who progressed after chemotherapy. The results show that immunotherapy combined with chemotherapy significantly improved the ORR and PFS compared to chemotherapy alone [14]. In the KEYNOTE-024 clinical study, 
pembrolizumab was used to treat patients with advanced lung cancer. Its PFS was 10.3 months, which was significantly longer than that of chemotherapy, and the risk of death was also reduced by $40 \%$, so it was approved by the FDA as the first-line treatment for patients with high PD-L1 expression in advanced NSCLC [15]. The KEYNOTE-010 [7] trial randomly assigned patients with PD-L1 expression $\geq 1 \%$ to be treated with different doses of pembrolizumab compared with docetaxel chemotherapy. Patients treated with $2 \mathrm{mg} / \mathrm{kg}$ and $10 \mathrm{mg} / \mathrm{kg}$ pembrolizumab at different doses had better OS (10.4 months vs. 12.7 months vs. 8.5 months) than those treated with chemotherapy. In 2016, atelizumab was approved by the FDA as a second-line treatment for advanced NSCLC. In 2018, the results of the IMpower131 study showed that regardless of PD-L1 expression, ateliizumab combined with chemotherapy as a first-line treatment can prolong the PFS of patients [16]. IMpower150[9] is a multicenter, open clinical study, which is the first phase III clinical trial that combines ICl with antiangiogenic therapy and chemotherapy for the first-line treatment of advanced nonsquamous NSCLC, including 1202 patients. The results showed that atelizumab combined with antiangiogenic therapy and chemotherapy provided significant PFS benefits in advanced NSCLC patients with EGFR or ALK mutations, low Teff expression, PD-L1 negativity, and liver metastasis compared with the control group (anti-angiogenesis combined chemotherapy), providing evidence of the effectiveness of the combination therapy, and the safety risks were consistent with previously reported single-agent therapy. Numerous clinical studies have shown that ICI therapy has a good therapeutic effect on NSCLC patients in the real world. In this study, the median PFS of the patients who took ICls as the first-line or second-line treatment was significantly longer than that in previous clinical trials.

Among the patients collected in our study, there were fewer EGFR mutation-positive patients, and the median PFS showed no significant difference between the EGFR mutations and the subgroup without mutations. Checkmate057[5] and KEYNOTE-010[7], these two clinical trial subgroup analysis results showed the comparison of ICl compared with chemotherapy for advanced NSCLC in the EGFR mutation group. There was no significant difference in OS, and EGFR mutation patients did not benefit from immunotherapy and were not sensitive to immunotherapy. The possible mechanism is that EGFR mutation is related to inhibiting the tumor microenvironment and reducing the tumor mutation burden [17]. In contrast, some scholars believe that it is impossible to generalize. In the subgroup analysis of the IMpower150 trial, 80 patients with EGFR-positive mutations who had previously failed EGFR-TKI treatment had a median PFS of 10.2 months after receiving immunotherapy combined with chemotherapy and antiangiogenesis therapy. This suggests that $\mathrm{ICl}$ combined with chemotherapy and antiangiogenic therapy may benefit patients with advanced NSCLC who have EGFR mutations[9]. Whether advanced lung cancer patients with EGFR mutations can benefit from immunotherapy has not been concluded at present, and more targeted clinical trial data are still needed.

In the treatment of lung cancer patients, they often face the use of hormones, such as treatment of brain metastases to reduce and prevent brain edema and improve tumor-related complications, the treatment of moderate to severe irAEs, and pretreatment before immunotherapy combined with chemotherapy. In this study, the patients with hormone interference mainly had previous autoimmune diseases and reduced irAEs. Some relevant clinical studies have confirmed that the application of hormones during ICI treatment may inhibit the antitumor efficacy of ICls [18-20]. The retrospective study conducted by Kathryn et al. [21] included 640 patients treated with $\mathrm{ICl}$ monotherapy in two cancer centers, of which 90 patients received hormone therapy ( $\geq 10 \mathrm{mg} / \mathrm{d}$ prednisone). The application of hormones affects the efficacy benefit of ICls and significantly reduces the PFS and OS of NSCLC patients. In the MSKCC cohort, hormone use was associated with a shorter median PFS (1.9 months vs 2.6 months) and a shorter median OS (5.4 months vs 12.1 months). In the GRCC cohort, the median PFS (1.7 months vs 1.8 months) and median OS (3.3 months vs 9.4 months) were also significantly reduced in those receiving

Page $15 / 23$ 
hormone therapy. The results of this study showed that the use of hormones shortened the median PFS, which was consistent with the results of previous retrospective studies.

Abx further interferes with the development of the system's immune response by affecting the function of T cells, changing the production of cytokines, and interfering with the role of dendritic cells (DCs) [22, 23]. In the study of immunotherapy, Abx is mainly used for infection of the lung, urinary system, skin, and other parts. Caicun Zhou et al. [24] reported in a previous study that 109 patients were included, among 57 patients who received ICI monotherapy and 19 patients who received ICI combined with antiangiogenic therapy. The data demonstrated a negative correlation of Abx treatment with PFS and OS. However, regarding the 33 patients who received ICls combined with chemotherapy, no significant difference in PFS and OS was observed between patients with or without Abx use. In our study, it was shown that regardless of ICI monotherapy or combination therapy, Abx affected the treatment effect and shortened PFS. The study of Hakozaki et al. [25] showed that NSCLC patients who received Abx before nivolumab treatment had a shorter median PFS and median OS than patients without Abx use (median PFS: 1.2 months vs. 4.4 months, median OS: 8.8 months vs. not reached), and the differences were statistically significant (all $\mathrm{P}<0.05$ ), but multivariate analysis showed that Abx treatment was not an independent predictor of PFS. A large retrospective study [26] included 291 patients with advanced tumors, 64 cases of NSCLC, 179 cases of melanoma, and 48 cases of renal cell carcinoma. Ninety-two patients were treated 2 weeks before and 6 weeks after receiving $\mathrm{ICI}$ treatment. Under the influence of controlling for other clinically relevant factors, the median OS of patients who received a single course of Abx treatment was shorter than that of the Abx-untreated group. Patients who received multiple courses or continuous use of Abx for more than 7 days had a shorter median OS and significantly reduced PFS. The study emphasizes that patients with multiple or longterm use of Abx have worse ICI treatment effects and aims to advocate that we use Abx with caution in the clinic. Our research also confirmed that Abx will affect the efficacy of ICls regardless of whether ICls are first-line or second-line treatment, and it was speculated that the length of Abx treatment was negatively correlated with the length of the median PFS. However, the effect of Abx treatment on the efficacy of ICls remains to be further evaluated.

PD-L1 expression is a marker related to the efficacy of first-line immunotherapy recommended by the NCCN guidelines. KEYNOTE-024 [4] is a comparative study of the efficacy of pembrolizumab treatment and standard platinum-containing chemotherapy for initially treated advanced NSCLC. The study included patients with PD-L1 expression $\geq 50 \%$. Compared with the chemotherapy group, the immunotherapy group had a higher ORR, longer PFS (10.3 months vs 6.0 months), longer OS (30.0 months vs 14.2 months), and a lower incidence of adverse reactions (73.4\% vs $90 \%$ ). The results of the controlled study of KEYNOTE-024 led the FDA to approve the treatment of pembrolizumab as the first-line treatment for NSCLC patients with positive PD-L1 expression (TPS $\geq 50 \%$ ). However, IMpower132[27] and KEYNOTE-189[8] compared the efficacy of immunotherapy combined with chemotherapy versus chemotherapy in patients with advanced NSCLC, and the results showed that immunotherapy improved PFS and OS regardless of PD-L1 expression. In the study using ICls as second-line treatment, KEYNOTE-010[7] was the first prospective study to demonstrate that PD-L1 expression is an important biomarker for predicting the efficacy of immunotherapy. A total of 1034 patients who had previously received chemotherapy were included in the phase III clinical study of NSCLC patients. According to the expression status of PD-L1, the expression of PD-L1 in all patients was $>1 \%$, and the results showed that the therapeutic efficacy of different doses of immunotherapy was better than that of patients receiving chemotherapy, and there was no OS benefit. For NSCLC patients with PD-L1 expression levels $\geq 50 \%$, the OS and median PFS of patients receiving immunotherapy were significantly longer than those receiving chemotherapy. For NSCLC patients with PD-L1

Page $16 / 23$ 
expression levels $<50 \%$, there was no statistically significant difference in OS between immunotherapy and chemotherapy $(P>0.05)$. These results indicated that the OS and PFS of patients with high PD-L1 expression were prolonged compared with those with low PD-L1 expression in second-line treatment. However, for patients with advanced NSCLC included in CheckMate 017[28] and OAK [29] who received second-line immunotherapy compared with chemotherapy, the level of PD-L1 expression could not predict the efficacy, and immunotherapy had advantages in improving PFS and OS. In the second-line immunotherapy of patients with advanced lung cancer, the current guidelines do not recommend PD-L1 detection as a basis for the guidance of treatment. The level of PD-L1 expression is the main factor predicting the efficacy of second-line immunotherapy and remains controversial. Compared with the second-line population, the median PFS of the population who used ICls as the first-line treatment in this study was prolonged. The analysis of this study showed that the median PFS with PD-L1 expression $\geq 50 \%$ was better than $1-49 \%$ and $<1 \%$ of patients in the treatment group, and there was a significant difference. Both this study and KEYNOTE-010 suggest that patients with high PD-L1 expression may be more likely to benefit from ICl treatment.

Since the advent of immunotherapy in the 1990s, it has made a breakthrough in the field of cancer treatment. The FDA and the National Medical Products Administration (NMPA) approved the first ICl for lung cancer treatment in 2015 and 2018. At present, an increasing number of ICls have been approved for lung cancer indications in China, and the application of immunotherapy in the field of lung cancer treatment has gradually become popular. Pembrolizumab is the first PD-1 antibody drug approved by the FDA. It was first used in the treatment of patients with advanced or unresectable malignant melanoma, but its potential in treating lung cancer was recently identified. At the American Society of Clinical Oncology (ASCO) annual meeting in May 2019, the first trial to enter the clinic, KeyNOTE-001, officially presented long-term efficacy results for pembrolizumab in advanced NSCLC patients, which is the longest follow-up study of pembrolizumab in patients with advanced NSCLC thus far [30]. A total of 550 lung cancer patients participated, with a 5 -year survival rate of first-line treatment patients of $23.2 \%$ and a 5 -year survival rate of second-line treatment patients of $15.5 \%$. Subsequent KEYNOTE-024 and KEYNOTE047 studies confirmed that pembrolizumab could prolong PFS and OS regardless of whether it was a single agent or combined with chemotherapy.

Although ICls have been widely used in clinical practice, two domestic immunologic drugs, sintilimab and toripalimab, have not yet been approved for the treatment of lung cancer. At present, the population with optimal benefit has not been selected, and large-scale clinical trials are still needed to explore the biomarkers of immunotherapy and the best drug combination therapy mode. In December 2018, based on the results of the ORIENT-1 study, sintilimab was officially approved by the NMPA for the treatment of patients with relapsed or refractory classical Hodgkin's lymphoma after at least second-line systemic chemotherapy. The ORIENT-11 study [31] is a randomized, double-blind, phase III controlled clinical trial evaluating the efficacy and safety of sintilimab or placebo in combination with pemetrexed and platinum for first-line treatment in advanced or relapsed nonsquamous NSCLC. The primary endpoint was PFS, and the secondary endpoints included OS and safety. As of November 2019, the study included a total of 397 patients. The median PFS of the experimental group was significantly longer than that of the placebo group (8.9 months vs 5.0 months), and the ORR of the experimental group was $51.9 \%$, which was also significantly higher than that of the placebo group (29.8\%). The median OS results of the two groups of patients were not yet reached, but patients in the sintilimab group had certain advantages. In each key clinical subgroup, sintilimab treatment had significant benefits and good safety. With the launch of toripalimab in February 2019, it was the first self-developed and marketed PD-1 inhibitor in China, benefiting some domestic patients with advanced tumors. Currently, the only approved indication is the second-line

Page $17 / 23$ 
treatment of advanced melanoma. In the case of poor efficacy of immune monotherapy and TK I combined immunotherapy, the CT18 study is the first domestic prospective phase II clinical study for Chinese patients with EGFR mutations and explores the efficacy and safety of toripalimab combined with chemotherapy for EGFR mutation-positive T790 M-negative advanced NSCLC patients who failed TK I treatment and had an ORR of 50.0\% and a median PFS of 7.0 months [32]. The study showed that toripalimab combined with chemotherapy for second-line treatment in patients with EGFR mutation-positive advanced NSCLC has a good curative effect and is safe and controllable, which is expected to become a new second-line treatment option for patients with EGFR mutation-positive advanced NSCLC. At present, a number of phase I, II, and III clinical trials of sintilimab and toripalimab for the treatment of lung cancer are advancing rapidly, which shows the contribution of innovative Chinese drugs in the field of tumor immunotherapy. There is also anticipation for an early approval date for the use of this treatment in lung cancer.

When considering the efficacy of drugs, safety is also one of the main clinical considerations. With the development of an increasing number of clinical trials, the adverse reactions of chemotherapy and immunotherapy have received increasing attention from researchers. Common adverse reactions caused by chemotherapy mainly include fatigue, nausea, vomiting, and bone marrow suppression. ICls act on immune cells, causing some normal cells of the body to be attacked to produce irAEs, which is essentially an inflammatory reaction caused by excessive activation of the immune system [33]. IrAEs can affect multiple systems, the most common of which are the skin, lungs, gastrointestinal tract, and endocrine system, and relatively rare occurrence sites are the nervous system and cardiovascular system [34,35]. Most irAEs have mild symptoms, but myocarditis, pneumonia, hepatitis, and neurotoxicity may be fatal, requiring clinical vigilance and attention [36, 37]. In the course of treatment, new respiratory symptoms, such as cough, chest tightness, and wheezing, should be suspected of pneumonia. The main imaging manifestations are ground-glass changes [38, 39].

In this study, the incidence of immune-related pneumonia was $11.1 \%$. Eleven patients developed immune-related pneumonia $\geq 3$ grade, $\mathrm{ICl}$ discontinuation and hormone therapy. Ten patients improved after treatment, and one patient died of the infection associated with immunosuppression. The patient received 10 cycles of sintilimab. At the end of the treatment, he developed cough, chest tightness, and repeated fever. Re-examination of chest CT revealed pneumonia, combined with multiple serous effusions and gastrointestinal hemorrhage, although he was given ventilator support and anti-infection, hormone and symptomatic treatment and eventually died of severe lung infection and respiratory failure. Two patients who received sintilimab and camrelizumab developed liver damage, continued increases in transaminase, combined with ascites, and later developed hepatic encephalopathy and eventually died due to disease progression, liver failure, and respiratory failure.

Immune-related myocarditis is a rare but relatively fatal irAE that manifests as increased troponin, creatine kinase, and BNP [40]. The incidence in this study was $3.5 \%$, one patient developed myocarditis after two cycles of treatment with camrelizumab, and troponin continued to increase. After stopping the drug, hormone therapy was still ineffective. Finally, the patient died of extensive myocardial injury. Myocarditis often has an early onset but has a high risk of death and should be identified and treated as soon as possible [41]. The incidence of irAEs is usually low, but once they cause adverse reactions of important organs, they may seriously endanger the lives of patients. The research data for irAEs remain incomplete, and there is a lack of effective methods to predict and screen patients with severe irAEs. In clinical work, we should detect and deal with irAEs as early as possible, cooperate with multiple disciplines, minimize risks, and strive to ensure the best benefit for patients. 


\section{Conclusion}

At present, the survival period of patients with advanced lung cancer is still very short, and immunotherapy has brought good news to patients, but the current understanding and research on immunotherapy is still the tip of the iceberg. How to choose the best combination therapy drug plan, how to screen the patient groups that may benefit the most, and how to choose evaluation indicators that are effective for prognosis all require future research and trials.

\section{Declarations}

\section{Ethics approval and consent to participate}

This study was approved by the Medical Ethics Committee in Weihai Municipal Hospital of Shandong Province. All patients provided written informed consent. This study was conducted in accordance with the World Medical Association Declaration of Helsinki, Good Clinical Practices, and local ethical/legal requirements

\section{Consent for publication}

Not applicable.

\section{Availability of data and materials}

The datasets generated and/or analysed during the current study are not publicly available due these data will be prepared for further studies, we will release relevant data when all the studies are completed, but are available from the corresponding author on reasonable request. To request data please contact Prof. Yu Li (email:

qlliyures@163.com).

\section{Competing interests}

The authors declare that they have no competing interests.

\section{Funding}

Not applicable.

\section{Authors' contributions}

MS and $\mathrm{HJ}$ drafted the manuscript. YL and MS were involved in conception and design. MS and HJ were involved in analysis and interpretation of data. MS, NX, PJ and TQ were involved in data collection. All authors contributed in review, revise and approve the manuscript; and agree to be accountable to all aspects of the work.

\section{Acknowledgments}

We express our gratitude to all the patients participated in this retrospective study. We are grateful to all the doctors at the participating institutions.

\section{References}


1. QIU L, ZHAO X, SHI W, et al. Real-world treatment efficacy of anti-programmed death-1 combined with antiangiogenesis therapy in non-small cell lung cancer patients. Medicine. 2020; 99:24.

2. SIEGEL R L, MILLER K D, JEMAL A. Cancer statistics, 2016. CA: a cancer journal for clinicians. 2016; 66(1): 7-30.

3. BUSTAMANTE ALVAREZ J G, GONZÁLEZ-CAO M, KARACHALIOU N, et al. Advances in immunotherapy for treatment of lung cancer. Cancer biology \& medicine. 2015; 12(3): 209-22.

4. RECK M, RODRÍGUEZ-ABREU D, ROBINSON A G, et al. Pembrolizumab versus Chemotherapy for PD-L1-Positive Non-Small-Cell Lung Cancer. The New England journal of medicine. 2016; 375(19): 1823-33.

5. BORGHAEI H, PAZ-ARES L, HORN L, et al. Nivolumab versus Docetaxel in Advanced Nonsquamous Non-SmallCell Lung Cancer. The New England journal of medicine. 2015; 373(17): 1627-39.

6. GARON E B, RIZVI N A, HUI R, et al. Pembrolizumab for the treatment of non-small-cell lung cancer. The New England journal of medicine. 2015; 372(21): 2018-28.

7. HERBST R S, BAAS P, KIM D W, et al. Pembrolizumab versus docetaxel for previously treated, PD-L1-positive, advanced non-small-cell lung cancer (KEYNOTE-010): a randomised controlled trial. Lancet. 2016; 387(10027): 1540-50.

8. GANDHI L, RODRÍGUEZ-ABREU D, GADGEEL S, et al. Pembrolizumab plus Chemotherapy in Metastatic NonSmall-Cell Lung Cancer. The New England journal of medicine. 2018; 378(22): 2078-92.

9. SOCINSKI M A, JOTTE R M, CAPPUZZO F, et al. Atezolizumab for First-Line Treatment of Metastatic Nonsquamous NSCLC. The New England journal of medicine. 2018; 378(24): 2288-301.

10.GADGEEL S M, LUKAS R V, GOLDSCHMIDT J, et al. Atezolizumab in patients with advanced non-small cell lung cancer and history of asymptomatic, treated brain metastases: Exploratory analyses of the phase III OAK study. Lung cancer. 2019; 128(105-12.

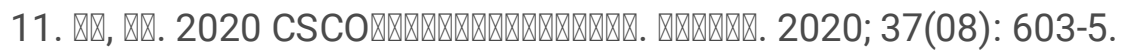

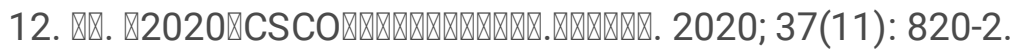

13. LIU B, SONG Y, LIU D. Recent development in clinical applications of PD-1 and PD-L1 antibodies for cancer immunotherapy. Journal of hematology \& oncology. 2017; 10(1): 174.

14. ARRIETA O, BARRÓN F, RAMÍREZ-TIRADO L A, et al. Efficacy and Safety of Pembrolizumab Plus Docetaxel vs Docetaxel Alone in Patients With Previously Treated Advanced Non-Small Cell Lung Cancer: The PROLUNG Phase 2 Randomized Clinical Trial. JAMA oncology. 2020; 6(6): 856-64.

15. RECK M, RODRÍGUEZ-ABREU D, ROBINSON A G, et al. Updated Analysis of KEYNOTE-024: Pembrolizumab Versus Platinum-Based Chemotherapy for Advanced Non-Small-Cell Lung Cancer With PD-L1 Tumor Proportion Score of $50 \%$ or Greater. Journal of clinical oncology. 2019; 37(7): 537-46.

16. ADRIANZEN HERRERA D, ASHAI N, PEREZ-SOLER R, et al. Nanoparticle albumin bound-paclitaxel for treatment of advanced non-small cell lung cancer: an evaluation of the clinical evidence. Expert opinion on 
pharmacotherapy. 2019; 20(1): 95-102.

17. HARATANI K, HAYASHI H, TANAKA T, et al. Tumor immune microenvironment and nivolumab efficacy in EGFR mutation-positive non-small-cell lung cancer based on T790M status after disease progression during EGFR-TKI treatment. Annals of oncology. 2017; 28(7): 1532-9.

18. RYKEN T C, MCDERMOTT M, ROBINSON P D, et al. The role of steroids in the management of brain metastases: a systematic review and evidence-based clinical practice guideline. Journal of neuro-oncology. 2010; 96(1): 103-14.

19. PAULSEN O, KLEPSTAD P, ROSLAND J H, et al. Efficacy of methylprednisolone on pain, fatigue, and appetite loss in patients with advanced cancer using opioids: a randomized, placebo-controlled, double-blind trial. Journal of clinical oncology. 2014; 32(29): 3221-8.

20. PUZANOV I, DIAB A, ABDALLAH K, et al. Managing toxicities associated with immune checkpoint inhibitors: consensus recommendations from the Society for Immunotherapy of Cancer (SITC) Toxicity Management Working Group. Journal for immunotherapy of cancer. 2017; 5(1): 95.

21. ARBOUR K C, MEZQUITA L, LONG N, et al. Impact of Baseline Steroids on Efficacy of Programmed Cell Death-1 and Programmed Death-Ligand 1 Blockade in Patients With Non-Small-Cell Lung Cancer. Journal of clinical oncology. 2018; 36(28): 2872-8.

22. ISMAIL A S, SEVERSON K M, VAISHNAVA S, et al. Gammadelta intraepithelial lymphocytes are essential mediators of host-microbial homeostasis at the intestinal mucosal surface. Proceedings of the National Academy of Sciences of the United States of America. 2011; 108(21): 8743-8.

23. ATARASHI K, TANOUE T, SHIMA T, et al. Induction of colonic regulatory T cells by indigenous Clostridium species. Science. 2011; 331(6015): 337-41.

24. ZHAO S, GAO G, LI W, et al. Antibiotics are associated with attenuated efficacy of anti-PD-1/PD-L1 therapies in Chinese patients with advanced non-small cell lung cancer. Lung cancer. 2019; 130(10-7.

25. HAKOZAKI T, OKUMA Y, OMORI M, et al. Impact of prior antibiotic use on the efficacy of nivolumab for nonsmall cell lung cancer. Oncology letters. 2019; 17(3): 2946-52.

26. TINSLEY N, ZHOU C, TAN G, et al. Cumulative Antibiotic Use Significantly Decreases Efficacy of Checkpoint Inhibitors in Patients with Advanced Cancer. The oncologist. 2020; 25(1): 55-63.

27. NISHIO M, BARLESI F, WEST H, et al. Atezolizumab Plus Chemotherapy for First-Line Treatment of Nonsquamous NSCLC: Results From the Randomized Phase 3 IMpower132 Trial. Journal of thoracic oncology. $2021 ; 16(4): 653-64$.

28. BRAHMER J, RECKAMP K L, BAAS P, et al. Nivolumab versus Docetaxel in Advanced Squamous-Cell NonSmall-Cell Lung Cancer. The New England journal of medicine. 2015; 373(2): 123-35.

29. RITTMEYER A, BARLESI F, WATERKAMP D, et al. Atezolizumab versus docetaxel in patients with previously treated non-small-cell lung cancer (OAK): a phase 3, open-label, multicentre randomised controlled trial Lancet. 2017; 389(10066): 255-65.

Page $21 / 23$ 
30. GARON E B, HELLMANN M D, RIZVI N A, et al. Five-Year Overall Survival for Patients With Advanced NonSmall-Cell Lung Cancer Treated With Pembrolizumab: Results From the Phase I KEYNOTE-001 Study. Journal of clinical oncology. 2019;37(28): 2518-27.

31. YANG Y, ZHOU H, ZHANG L. Response to Letter to the Editor: Efficacy and Safety of Sintilimab Plus Pemetrexed and Platinum as First-Line Treatment for Locally Advanced or Metastatic Nonsquamous NSCLC: A Randomized, Double-Blind, Phase 3 Study (ORIENT-11). Journal of thoracic oncology. 2020, 15(12): 191-2.

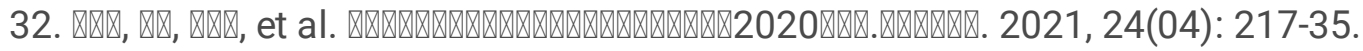

33. DAS S, JOHNSON D B. Immune-related adverse events and anti-tumor efficacy of immune checkpoint inhibitors. Journal for immunotherapy of cancer. 2019; 7(1): 306.

34. KHAN Z, HAMMER C, GUARDINO E, et al. Mechanisms of immune-related adverse events associated with immune checkpoint blockade: using germline genetics to develop a personalized approach. Genome medicine. 2019; 11(1): 39.

35. WANG Y, ZHOU S, YANG F, et al. Treatment-Related Adverse Events of PD-1 and PD-L1 Inhibitors in Clinical Trials: A Systematic Review and Meta-analysis. JAMA oncology. 2019; 5(7): 1008-19.

36. WANG D Y, SALEM J E, COHEN J V, et al. Fatal Toxic Effects Associated With Immune Checkpoint Inhibitors: A Systematic Review and Meta-analysis. JAMA oncology. 2018; 4(12): 1721-8.

37. LYON A R, YOUSAF N, BATTISTI N M L, et al. Immune checkpoint inhibitors and cardiovascular toxicity. The Lancet Oncology. 2018; 19(9): 447-58.

38. NAIDOO J, WANG X, WOO K M, et al. Pneumonitis in Patients Treated With Anti-Programmed Death1/Programmed Death Ligand 1 Therapy. Journal of clinical oncology. 2017; 35(7): 709-17.

39. NISHINO M, CHAMBERS E S, CHONG C R, et al. Anti-PD-1 Inhibitor-Related Pneumonitis in Non-Small Cell Lung Cancer. Cancer immunology research. 2016; 4(4): 289-93.

40. BRUMBAUGH A D, NARURKAR R, PARIKH K, et al. Cardiac Immune-Related Adverse Events in Immune Checkpoint Inhibition Therapy. Cardiology in review. 2019; 27(2): 97-107.

41. MOSLEHI J J, SALEM J E, SOSMAN J A, et al. Increased reporting of fatal immune checkpoint inhibitorassociated myocarditis. Lancet. 2018; 391(10124): 933.

\section{Figures}




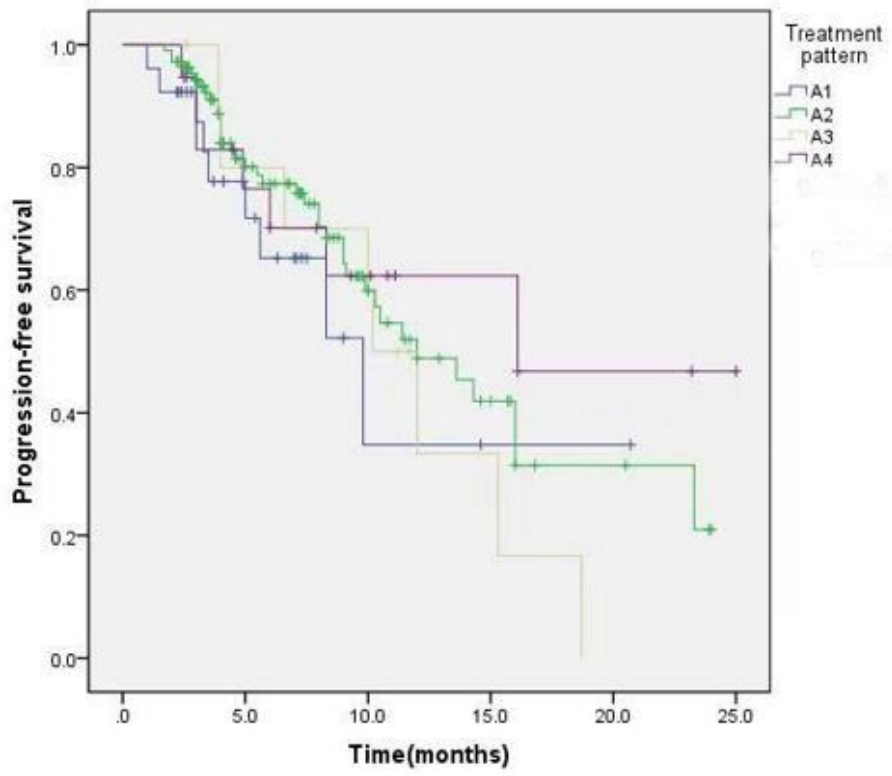

1 line treatment of PFS

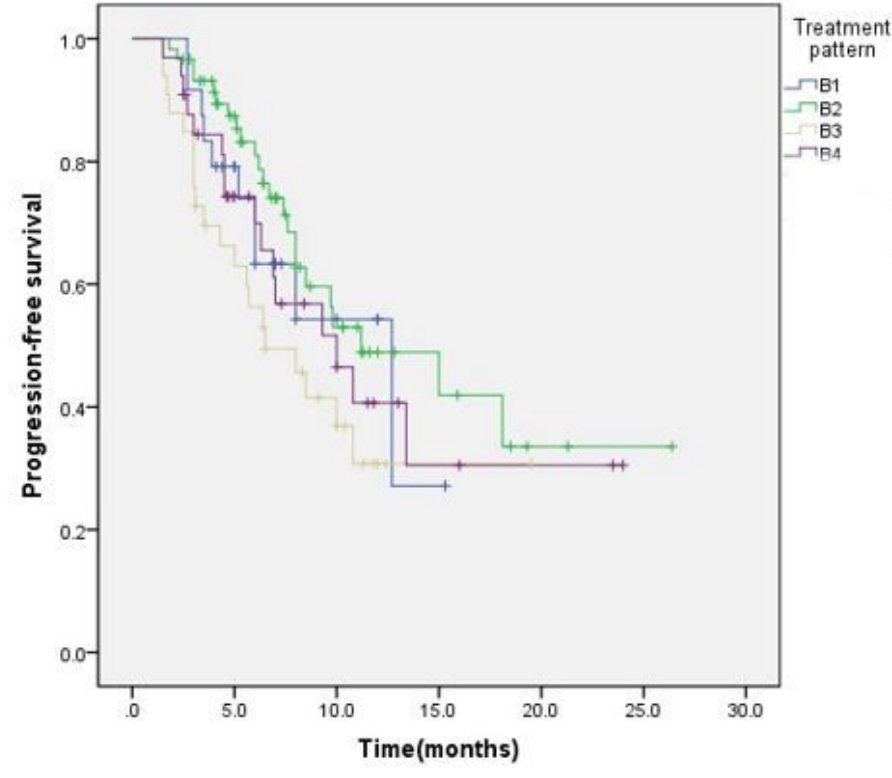

2 line treatment of PFS

Figure 1

Kaplan-Meier survival curve of PFS 